\title{
Educação para controle da asma
}

\author{
Education for asthma control
}

\author{
Leonardo Araujo Pinto \\ Pneumologista Pediátrico. Professor da Faculdade de Medicina da Pontifícia Universidade Católica do Rio Grande do Sul.
}

DESCRITORES: EDUCAÇÃO EM SAÚDE; ESTUDOS DE INTERVENÇÃO; ASMA; CRIANÇA; ADOLESCENTE.

KEY WORDS: HEALTH EDUCATION; INTERVENTION STUDIES; ASTHMA; CHILD; ADOLESCENT.

A asma afeta aproximadamente entre $5-20 \%$ da população mundial, demonstrando uma prevalência elevada e muito variável. No Brasil, estudos realizados com amostras de crianças em idade escolar demonstram taxas de prevalência que ficam próximas dos níveis mais elevados entre todos os países pesquisados $(20 \%$ da população pediátrica). ${ }^{1,2}$ Apesar de ser considerada uma das principais doenças crônicas da infância, apenas $50 \%$ das crianças acometidas realizam o tratamento de controle adequadamente. ${ }^{3}$ Estes dados demonstram a necessidade de programas de educação que possam contribuir para um aumento da aderência ao tratamento correto e do controle da doença.

$\mathrm{O}$ estudo de Cidade SF et al., ${ }^{4}$ publicado nesta edição da Scientia Medica, descreve as diferentes intervenções de educação para crianças com asma. Esta comparação entre os diversos métodos de intervenção pode contribuir para que os profissionais da saúde envolvidos escolham e desenvolvam intervenções eficazes no momento do planejamento de um programa de controle de asma.
Outro tópico de grande relevância, e também relacionado ao tema, são as intervenções de educação para médicos e profissionais de saúde. Quando observamos um problema de aderência ao tratamento, em geral, ocorre um déficit entre a informação que o médico deseja passar e a informação realmente compreendida pelos pais ou pacientes. Este "gap" não deve ser justificado, exclusivamente, pela dificuldade de entendimento dos pacientes, mas também pela capacidade dos profissionais de saúde de passarem a informação de forma eficaz.

Considerando o impacto abrangente da doença, que afeta todos os países e todas as faixas etárias, existe um grande avanço em termos de publicações originais referentes a novos tratamentos. Porém, a eficiência dessas intervenções novas, ou das já consolidadas, poderá ser comprometida se não houver medidas para aumentar a aderência ao tratamento. Essas medidas certamente passam pelas intervenções de educação para pacientes e profissionais de saúde.

\section{REFERÊNCIAS}

1. Barreto ML, Ribeiro-Silva R de C, Malta DC, Oliveira-Campos M, Andreazzi MA, Cruz AA. Prevalence of asthma symptoms among adolescents in Brazil: National Adolescent School-based Health Survey (PeNSE 2012). Rev Bras Epidemiol. 2014;17(Suppl 1):106-15.

2. Solé D, Filho NA, Sarinho ES, Camelo-Nunes IC, Barreto BA, Medeiros ML, Motta JM, Camargos PA, Mallol J, Gurgel R, de Andrade DM, Furlan FP, Silva AR, Cardozo C, Andrade C. Prevalence of asthma and allergic diseases in adolescents: nine-year follow-up study (2003-2012). J Pediatr (Rio J). 2014. doi: 10.1016/j.jped.2014.05.002. [Epub ahead of print].

3. Morton RW, Everard ML, Elphick HE. Adherence in childhood asthma: the elephant in the room. Arch Dis Child. 2014;99(10):949-53.

4. Cidade SF, Roncada C, Costa DD, Pitrez PM. Educação em asma: principais técnicas adotadas em programas de intervenção. Sci Med. 2014;24(3):297-306. 